\title{
Modeling of aqueous foam blast wave attenuation
}

\author{
E. Del Prete ${ }^{1, a}$, A. Chinnayya ${ }^{2}$, A. Hadjadj ${ }^{2}$, L. Domergue ${ }^{1}$, J.-F. Haas ${ }^{1}$, and B. Imbert ${ }^{1}$ \\ 1 CEA, DAM, DIF, 91297 Arpajon, France \\ 2 CORIA CNRS UMR-6614, Site du Madrillet, 76801 Saint Etienne du Rouvray, France
}

\begin{abstract}
The use of aqueous foams enables the mitigation of blast waves induced by the explosion of energetic materials. The two-phase confinement gives rise to interphase interactions between the gaseous and liquid phases, which role have been emphasized in shock-tube studies with solid foams [1,2]. Multifluid formalism enables the thermomechanical disequilibria between phases to be taken into account. The flow model ensures the correct estimation of the acoustic impedance of the two-phase media. As for the numerical scheme, Riemann solvers are used to describe the microscopic fluid interactions, the summation of which provides the multiphase flux. The role of the different transfer mechanisms is evaluated in the case where the liquid ligaments of the foam matrix have been shattered into droplets by the shock impingement. Characteristics of blast waves in heterogeneous media leads to a decrease of overpressure. The numerical results have been compared favorably to experimental data $[3,4]$.
\end{abstract}

\section{Introduction}

Blast waves resulting from explosions in air can cause serious damages to structures and humain being located many charge radii from the explosion point of origin. When the detonation wave propagating through the condensed explosive reaches the explosive/air interface, an intense shock wave with order of hundred atmosphere pressure is propagated radially outwards through the air. It has been shown that the strength of the blast wave can be greatly attenuated by surrounding the explosive charge with aqueous foam [3]. Since over 50 years, several laboratories in the world conducted experimental campaigns to define the efficiency of aqueous foam to mitigate blast waves. The objective of this paper is to understand the underlying physics and to model the multiphase flow resulting from the complex blast wave/foam interaction.

An aqueous foam is a two-phase system in which gas cells are enclosed by liquid films. The gaseous phase is dispersed in bubbles, whereas the liquid phase is the continuous phase. The bubbles take the form of polyhedral cells (Fig. 1), with liquid surfaces meeting in lines and lines merging at vertices. The lines are called Borders Plateau, which are channels of finite width and where the liquid is mainly to be found. This topological description corresponds to a dry foam. As liquid volume fraction increases, Plateau Borders swell and the bubbles progressively recover their spherical shape. This corresponds to a wet foam. Any further increase of liquid will allow them to come apart and the foam becomes a bubbly liquid. To summarize the resulted spectrum of the aqueous two-phase medium, the following classification according to the value of the liquid volume fraction $\alpha_{1}$ can be made $[5,6]$ :

- dry foam: $\alpha_{1}<0.05$

- wet foam: $0.05<\alpha_{1}<0.36$

- bubbly liquid: $\alpha_{1}>0.36$.

\footnotetext{
a e-mail: emilie.delprete@cea.fr
}

This is an Open Access article distributed under the terms of the Creative Commons Attribution-Noncommercial License 3.0, which permits unrestricted use, distribution, and reproduction in any noncommercial medium, provided the original work is properly cited. 


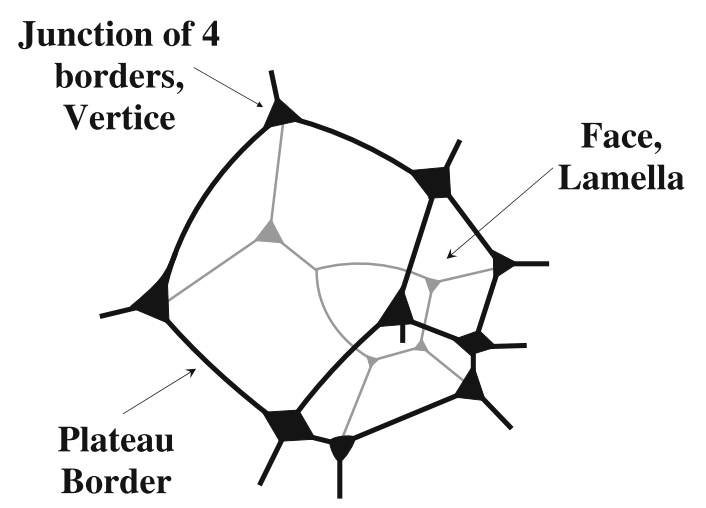

Fig. 1. Structure of a dry foam [7].

Aqueous foams are natural but metastable states. Under high pressure ratio shock wave impingement, the liquid matrix is likely to be shattered into more stable droplets [8]. This has lead to model the process of shock wave attenuation -in shock tubes, by a foam screen formed by a gaseous suspension of water droplets [9].

\section{Numerical modeling}

In this study, a two-fluid model is developed assuming a desequilibrium between pressure, velocity and temperature. Indeed, the passage of shock wave over a two-phase system will cause the two phases to be brought to different mechanical and thermodynamic states, due to their impedance contrast. Relaxation processes will attempt to edge the phases towards equilibrium. The post-shock states will then relax to the same thermo-mechanical state at the end of a relaxation zone. The model is derived based on the eulerian balances for the volume fraction, mass, momentum and total energy of each phase. Under a compact form, the two-fluid model can be written in spherical coordinate $r$ :

$$
\frac{\partial(\alpha W)_{k}}{\partial t}+\frac{\partial(\alpha F)_{k}}{\partial r}=F_{k}^{l a g} \frac{\partial \alpha_{k}}{\partial r}+S_{g e o m, k}+S_{d, k}
$$

where $\alpha_{k}, \rho_{k}, u_{k}, E_{k}, H_{k}, P_{k}$ are respectively the volume fraction, density, velocity, total energy, total enthalpy and pressure of each phase. $W=(1, \rho, \rho u, \rho E)$ is the fluid conversative variables, $F=\left(0, \rho u, \rho u^{2}+P, \rho E u+P u\right)$ the fluid eulerian flux and $F^{l a g}=\left(-u_{i}, 0, P_{i}, P_{i} u_{i}\right)$ the fluid lagrangian flux. Each phase is governed by its own equation of state: perfect gas law for the gas phase and stiffened gas for the liquid phase. The interfacial variables $P_{i}, u_{i}$ are issued from the homogenization method Discrete Equation Method (DEM) [10]. They play a key role on material interfaces which separate non-miscible fluids and enable the interface conditions - velocity and pressure uniformity across an interface, to be satisfied by the model. $S_{\text {geom }}$ stands for the spherical divergence and $S_{d}$ accounts for the momentum and energy exchanges between phases.

The pressure relaxation rate is infinite [11]. The momentum interphase interaction is represented by the drag force of the gaseous phase on the liquid [12]. Energy exchange is associated to heat transfer. These constitutive phenomenological relations enable to close the dissipative source terms $S_{d}$.

The derivative terms are at the origin of the hyperbolicity of the system and control the wave propagation in the two-fluid media. This model is unconditionnally hyperbolic. An asymptotic derivation towards a one velocity one pressure hyperbolic model can be achieved. Its analysis then shows that the sound speed of the two-phase mixture is the non-monotonic sound speed of Wood/Wallis. The latter speed is below the sound speed of pure fluids, due to the contrast of acoustic properties. Indeed, mixture density is close to the apparent density of liquid and compressibility to that of the gaseous phase. With the increase of liquid volume fraction, the effective two-phase media becomes impenetrable to pressure wave propagation. 


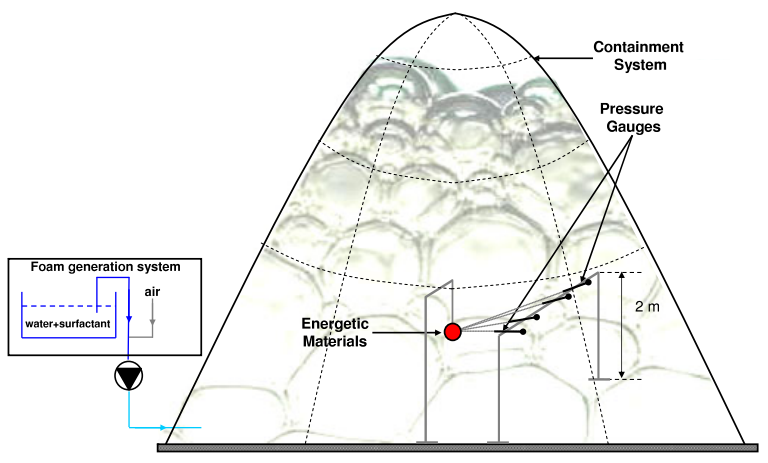

Fig. 2. Experimental configuration of CEA campaign [4].

The resolution of our two-fluid model by the DEM proceeds in several steps. The first step is the fluid selection by an indicative function. Then the integration of the variables in a specific control volume follows. The microscopic contacts between immiscible fluids and their corresponding lengths have to be determined. Finally, the fluxes that embody the fluid-contacts are sampled, then weighted with the corresponding length contact to provide the multiphase flux.

\section{Experimental setup}

The Fig. 2 depicts the experimental configuration which has been designed with a particular attention paid to the sphericity of the air blast wave free of any obstacle [4]. The High Explosive (HE) packed in a spherical enveloppe was suspended at a height of 2 meters from the ground. The ignition was made at its center. The pressure gauges were placed at the same height as the HE, so that the reflected Mach waves from the ground do not alter the duration of the positive impulse. They are placed on a gantry and oriented towards the center of the explosive: the membranes that allow the measurements are placed in the fluid flow direction and not frontally with the shock wave. In the first set of experiments, a charge of $6.6 \mathrm{~kg}$ of Plastrite -Pentrite based was detonated in air. The seven PCB gauges were placed between 2 and 5 meters from the detonator. After proper calibration of the electric signals, the evolution of the maximum overpressure, as a function of reduced radius, was found to follow the airblast tables of Kinney-Graham [13] with a relative TNT effectiveness factor of 1.27. Then, a second set of experiments was done with the same mass of energetic material, which was confined with an aqueous foam of expansion ratio of $1: 125$.

\section{Numerical results and discussion}

The mesh contains 1500 cells for a computational domain of $3.5 \mathrm{~m} \cdot \mathrm{kg}^{-1 / 3}$. The droplet size of the fragmented aqueous foam is chosen to be $50 \mu \mathrm{m}$ and is about the same order of magnitude as the Border Plateau radius. The aqueous foam is considered to be in atmospherical conditions at sea level. The volume fraction is the inverse of the expansion ratio. As for the initial conditions for detonation products, the constant volume approach has been used. The initial density of the HE is $1654 \mathrm{~kg} . \mathrm{m}^{-3}$ and the detonation energy is $4.517 \mathrm{MJ}_{\mathrm{kg}}{ }^{-1}$. The other thermodynamic variables are deduced from the equations of state.

\subsection{Overpressure results}

At first, the experimental overpressures have been compared with the tables given in [13] and reported in Fig. 3. There is a good agreement between the two sets of data, with a relative effectiveness factor of 1.27 for the Plastrite energetic material. It can be seen that for the mean field, there is also a good 


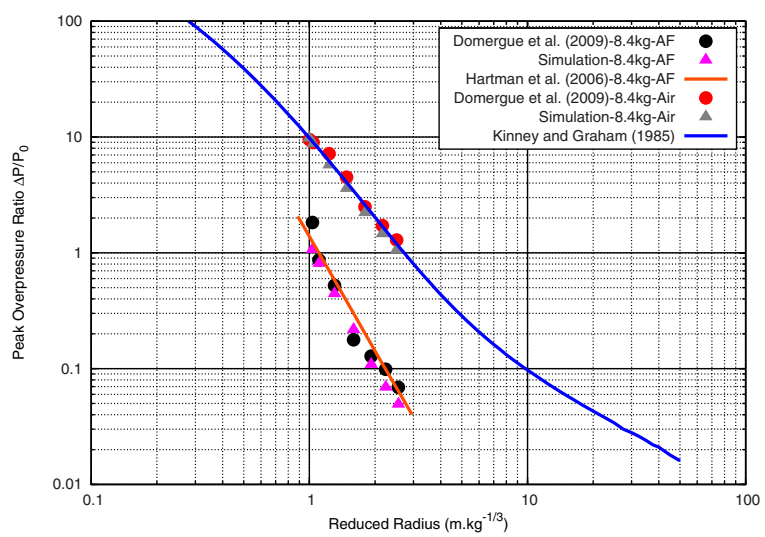

Fig. 3. Overpressure ratio comparison between an air explosion and an aqueous foam confined explosion.

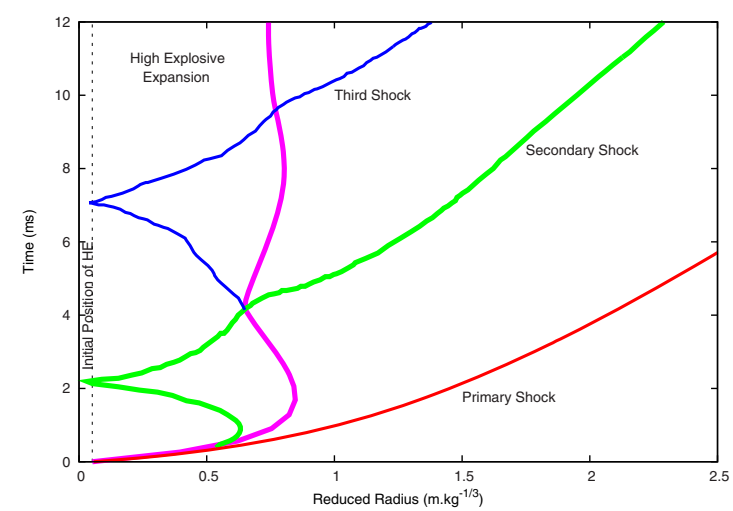

Fig. 4. Wave diagram of shocks and fireball interface - in air.

agreement between the numerical results and the experimental data. In the studied range, the blast wave overpressure decreases with the reduced radius at approximately power two. Not shown here, the positive impulse as well as the time of arrival and the shock velocity compare also favorably with experiments.

Regarding the overpressures when the HE is confined by the aqueous foam, the experimental results are fitted by the empirical relation derived from [3]. The numerical results show also a good agreement. The ratio of overpressures in the air over those in foams increases from approximately 1 decade to 1.5 decade in the range of reduced radius of 1 to 3 . This clearly demonstrates the blast mitigation capabilities of aqueous foams.

From the numerical point of view, the post-shock total energy can be estimated via linearized Riemann invariants and can be shown to decrease with an increase of the acoustic impedance of the confining medium. This energy radiates spherically, and will result in a lower overpressure. Moreover, a two-phase shock is a composite shock, which consists of a shock wave followed by a relaxation zone. The spherical rarefaction will thus tend to smooth the peak overpressure. In addition, the compressibility of the two-phase confinement is mainly driven by the gaseous phase. The liquid phase tends to store the kinetic energy given by the spherical shock wave. Decreasing the temperature of the gas will also decrease the level of the overpressure.

\subsection{Wave diagram}

The fireball position, the main shock as well as the subsequent ones are shown in Fig. 4. As the acoustic impedance of air is very low compared to that of the detonation products, the shock wave in 


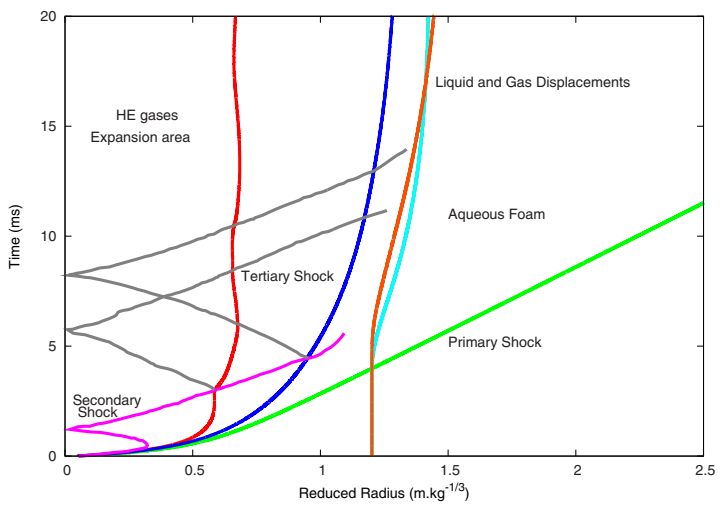

Fig. 5. Wave diagram of shocks and fireball interface - in aqueous foam.

air starts with an initial strength well below that of the high pressure volume and very shortly decays with the spherical divergence. This shock is mainly responsible for the maximum overpressure seen by the pressure jauge. Between the outward moving shock and the inward rarefaction wave, a second shock develops and begins to grow inward from the fireball interface while the latter moves out in general expansion. This inward secondary shock then implodes and reflects on the origin. At that time, the fireball is ending its expansion and is beginning to shrink. The detonation products are still cooler than the air immediately outside. Thus the reflection from the secondary shock give rise to rarefaction waves. Like in the initial rarefaction waves, they are followed by a third shock. Then this complex shock inverts the fireball velocity abruptly. The succession of shocks continues in this fashion until the energy of the detonation products is dissipated. The origin of these inward shock lies in the necessity of a compression wave at the junction between a spherical rarefaction wave moving away from a spherical shock wave [14].

In the same way as previously, the wave diagram is presented in Fig. 5 in the case of aqueous foam confinement, for the different shocks as well as the liquid and gaseous interfaces. Indeed, the liquid phase has its own dynamics and own velocity. The same global features of inward and outward shocks are found as compared to the air configuration. However, there is another interface. During the first phase of expansion, the liquid and gas fluids of the aqueous foam have approximately the same velocity. Then the gas slows down with spherical divergence. However liquid inertia is greater than the gas. So its final abscissa is greater than the gas. The liquid is then projected at a greater distance than the gas. The aqueous foam and the fireball are thus separated. The secondary shock reflects on this liquid interface. A weak compression wave is transmitted to the foam and a shock get reflected in the gaseous phase which lies between the aqueous foam and the fireball. A more complex dynamic of reflected and transmitted shocks will ensue with the presence of this gap. The final position of the fireball in the air configuration is slightly greater than in the foam configuration, as the foam density is greater. Moreover, the dynamics is also different. In air, after an initial phase of expansion, the fireball shrinks before being impacted by the secondary shock, which inverts its velocity. In the second case, as the acoustic impedance of the foam is greater, the initial velocity of the fireball is less. The secondary shock impacts the interface at the same time as the contracting phase begins to occur. In addition, the time at which the secondary shock implodes on the origin is shorter because the rarefaction waves are weaker in the first instants. As a consequence, the oscillating character of the fireball in the air will be more marked than in the confinement case in the first period of expansionshrinking. Then the internal wave pattern into the gap, that is the multiple goings and comings of the different compression/expansion waves into the gap will smoothen the evolution of the position of the fireball towards its end position.

The dynamics of the liquid and gaseous phase is also different in the far field. A pair of Lagrangian probes for the liquid and gas, respectively in blue turquoise and red were initially placed at a reduced radius of $1.3 \mathrm{~m} \cdot \mathrm{kg}^{-1 / 3}$. After passage of the primary shock wave, the gas is put in motion. The liquid will then be put into motion by the gas phase. So after an initial expansion phase, the gas velocity 
will fall during the shrinking phase. However, due to the greater liquid inertia, the distance travelled by droplets will be in the end greater than the gas particle. The trajectories of the liquid and gas can clearly be seen to be intertwined. The velocity of the fireball in the first stage of expansion is lowered with the aqueous foam confinement. It acts as a piston on its environnment. The time during which this piston is maintained is less. This will result in a lower overpressure field. In addition, the gap between the aqueous foam and the fireball acts as a buffer.

\section{Conclusion}

A multifluid formalism is used to describe an aqueous foam as a dense two-phase medium. After shock impingement, the initially encapsulated gas pores and liquid ligaments become respectively the carrier phase and the dispersed phase composed of liquid droplets. Conventional constitutive relations for gas-droplets have been used to describe the inter-phase exchanges within a multifluid approach. A good agreement between the numerical results and the experimental data is obtained.

Moreover, the particularities of the two-phase wave diagram in comparison to the air configuration have been discussed. A gap is created between the fireball and the two-phase media. Liquid and gas paths are intertwined. This emphasizes the non-equilibrium effect of the aqueous foam under shock impingement and its blast mitigation capabilities.

\section{References}

1. Baer M.R., Shock Waves 2, (1992) 121-124

2. Seitz M.W., Skews B.W., Shock Waves 15, (2006) 177-197

3. Hartman W. et al., SANDIA Report (SAND2006-0533, 2006)

4. Domergue L. et al., Safety and Security Engineering III, (2009)

5. Lorenceau E. et al., Congrès Français de Mécanique, Grenoble, France (2007)

6. Britan A. et al., Colloids ans Surfaces A: Physicochemical and Engineering Aspects 309, (2006) $137-150$

7. Weaire D., Hutzler S., The Physics of Foams (Oxford University Press, 1999)

8. Britan A.B et al., Combustion, Explosion and Shock Waves 28, (1992) 550-557

9. Britan A. et al., Combustion, Explosion and Shock Waves 30, (1994) 384-396

10. Chinnayya A. et al., Journal of Computational Physics 196, (2006) 490-538

11. Lallemand M.-H. et al. International Journal for Numerical Methods in Fluids, 49-1, (2005) 1-56

12. Ortiz C.et al. International Journal of Multiphase Flow, 30-2, (2004), 217-224

13. Kinney G.F., Graham K.J., Explosive shocks in air (Springer-Verlag, 1985)

14. Brode H. L., Physics of fluids 2, (1959) 217-229 